\title{
In situ reduced MXene/AuNPs composite toward enhanced charging/discharging and specific capacitance
}

\author{
Zixiang ZHENG ${ }^{a, \dagger}$, Wei $\mathrm{WU}^{a, \dagger}$, Tao YANG ${ }^{a,{ }^{*}}$, Enhui $\mathrm{WANG}^{a}$, Zhentao $\mathrm{DU}^{b}$, \\ Xinmei HOU ${ }^{a, *}$, Tongxiang $\mathrm{LIANG}^{c}$, Hailong $\mathrm{WANG}^{d}$
}

${ }^{a}$ Beijing Advanced Innovation Center for Materials Genome Engineering, Collaborative Innovation Center of Steel Technology, University of Science and Technology Beijing, Beijing 100083, China

${ }^{b}$ MOE Key Laboratory of New Processing Technology for Non-ferrous Metals and Materials, Guangxi Key

Laboratory of Processing for Non-ferrous Metals and Featured Materials, Guangxi University, Nanning 530004, China

${ }^{c}$ School of Materials Science and Engineering, Jiangxi University of Science and Technology, Ganzhou 341000, China

${ }^{d}$ School of Materials Science Engineering, Zhengzhou University, Zhengzhou 450001, China

Received: February 4, 2021; Revised: April 28, 2021; Accepted: May 3, 2021

(C) The Author(s) 2021.

\begin{abstract}
In this work, gold nanoparticles (AuNPs) decorated $\mathrm{Ti}_{3} \mathrm{C}_{2} \mathrm{~T}_{x}$ nanosheets (MXene/AuNPs composite) are fabricated through a self-reduction reaction of $\mathrm{Ti}_{3} \mathrm{C}_{2} \mathrm{~T}_{x}$ nanosheets with $\mathrm{HAuCl}_{4}$ aqueous solution. The obtained composite is characterized as AuNPs with the diameter of about $23 \mathrm{~nm}$ uniformly dispersing on $\mathrm{Ti}_{3} \mathrm{C}_{2} \mathrm{~T}_{x}$ nanosheets without aggregation. The composite (MXene decorated on $4.8 \mathrm{wt} \%$ AuNPs) is further employed to construct supercapacitor for the first time with a higher specific capacitance of $278 \mathrm{~F} \cdot \mathrm{g}^{-1}$ at $5 \mathrm{mV} \cdot \mathrm{s}^{-1}$ than that of pure $\mathrm{Ti}_{3} \mathrm{C}_{2} \mathrm{~T}_{x}$ and $95 \%$ of cyclic stability after 10,000 cycles. Furthermore, MXene/AuNPs composite symmetric supercapacitor with filter paper as separator and $\mathrm{H}_{2} \mathrm{SO}_{4}$ as electrolyte, is assembled. The supercapacitor exhibits a high volumetric energy density of $8.82 \mathrm{Wh} \cdot \mathrm{L}^{-1}$ at a power density of $264.6 \mathrm{~W} \cdot \mathrm{L}^{-1}$ and ultrafast-charging/ discharging performance. It exhibits as a promising candidate applied in integrated and flexible supercapacitors.
\end{abstract}

Keywords: supercapacitors; MXene; gold nanoparticles (AuNPs); self-reduction; composite

\section{Introduction}

On account of excellent cycle stability, high rate of charge/discharge, and high-power density, supercapacitors

\footnotetext{
$\uparrow$ Zixiang Zheng and Wei Wu contributed equally to this work

* Corresponding authors.

E-mail: X. Hou, houxinmeiustb@ustb.edu.cn; T. Yang, yangtaoustb@ustb.edu.cn
}

have caught great attention during these years [1]. Now one of the key challenges in making supercapacitors is improving the energy density [2]. Researchers concentrate on exploring and developing the electrode materials with excellent performance, which are key ingredients for high performance supercapacitors [3]. Two-dimensional (2D) materials are among the most promising electrode materials for supercapacitors. Due to their high surface-area-to-volume ratios, 2D materials 
offer an abundance of active surface area for charge storage through electric double layer capacitance (EDLC) or fast surface redox reactions (pseudocapacitance) [4]. During the last decade, due to relatively better chemical stability, higher specific surface area and active surface sites, excellent hydrophilicity, and higher electrical conductivity, transition metal carbides and/or nitrides (MXene) as a novel family of 2D materials have been applied in different areas including energy storage, water desalination, catalysis, electromagnetic interference shielding, and transparent conductive films [5-7]. The general formula of MXenes is $\mathrm{M}_{n+1} \mathrm{X}_{n} \mathrm{~T}_{x}(n=1-3)$, where $\mathrm{M}$ represents a transition metal (such as $\mathrm{Sc}, \mathrm{Ti}$, and $\mathrm{Zr}$ ), $\mathrm{X}$ is carbon and/or nitrogen, and $\mathrm{T}$ stands for the surface termination (i.e., hydroxyl, oxygen, or fluorine) [5]. The earliest explored and the most widely applied MXene is $\mathrm{Ti}_{3} \mathrm{C}_{2} \mathrm{~T}_{x}$ which shows exceptional performances as a potential electrode material for supercapacitors [8].

However, due to the presence of van der Waals force, almost all of 2D materials, including MXene, have the inevitable problems of aggregation and self-restacking [9]. This problem prevents electrolyte penetration into layers, limits the ion transport, and reduces the active site in supercapacitors. Therefore the restacking has become a huge obstacle to ameliorate the performance of MXene [10]. In order to solve the problem, various interlayer spacers are introduced into 2D materials to prevent the stacking of them, which can accelerate electrolyte penetration and ion diffusion $[11,12]$. These include carbon nanomaterials such as carbon nanotubes (CNTs) [13] and graphene [14,15], conductive polymers such as polypyrrole (PPy) [16], poly(3,4-ethylenedioxythiophene) (PEDOT) [17], polyaniline (PANI) [18], and metal nanoparticles such as silver nanoparticles (AgNPs) [19] and gold nanoparticles (AuNPs) [11]. In particular, since AuNPs is one of the highest conductivity metals with large specific surface area and high stability at the nanoscale level, it can be available for high performance energy storage devices [20]. To the best of our ability, there are still no reports about $\mathrm{Ti}_{3} \mathrm{C}_{2} \mathrm{~T}_{x}$ nanosheets modified by AuNPs to improve the electrochemical properties.

In this work, AuNPs decorated $\mathrm{Ti}_{3} \mathrm{C}_{2} \mathrm{~T}_{x}$ nanosheets were fabricated through a self-reduction reaction of $\mathrm{Ti}_{3} \mathrm{C}_{2} \mathrm{~T}_{x}$ with $\mathrm{HAuCl}_{4}$ aqueous solution. By vacuum filtration of the mixture solution, freestanding composite films were fabricated. Then MXene/AuNPs composite was used as electrode material of supercapacitors for the first time. MXene/AuNPs electrodes showed significantly enhanced electrochemical property compared to the pure $\mathrm{Ti}_{3} \mathrm{C}_{2} \mathrm{~T}_{x}$ electrodes. The integrated device can power a red light emitting diode (LED), demonstrating its energy storage capacity.

\section{Experimental}

\section{1 Materials}

High-purity $\mathrm{Ti}_{3} \mathrm{AlC}_{2}$ MAX phase powders (99 wt\%) were supplied by Kaikai Materials Co., Ltd., China. $\mathrm{HAuCl}_{4}$ was purchased from Sinopharm Chemical Reagent Co., Ltd., China. Xinya Equipment Co., Ltd., China, supplied cellulose membrane with $0.44 \mu \mathrm{m}$ pore size and $\phi 50 \mathrm{~mm}$ in diameter. The coin cell (CR2032CASE-304Pt, coating Pt of the thickness of 300-500 $\AA$ ) was supplied by Hefei Kejing Materials Technology Co., Ltd., China.

\section{2 Preparation of MXene/AuNPs composite}

In view of the preparation of $\mathrm{Ti}_{3} \mathrm{C}_{2} \mathrm{~T}_{x}$, minimally intensive layer delamination (MILD) method was used [21]. Briefly, $12 \mathrm{M} \mathrm{HCl}$ of $40 \mathrm{~mL}$ solution and $\mathrm{LiF}$ of $2 \mathrm{~g}$ were mixed with stirring of $30 \mathrm{~min}$. After the powder was completely dissolved, $\mathrm{Ti}_{3} \mathrm{AlC}_{2}$ powders of $2 \mathrm{~g}$ were mixed slowly with the $\mathrm{LiF}-\mathrm{HCl}$ solution. The as-prepared solution was stirred with $500 \mathrm{rpm}$ for $24 \mathrm{~h}$ at $35{ }^{\circ} \mathrm{C}$. The suspension was completely transferred to a centrifuge tube. Then the etched powder was washed using deionized water to ensure the $\mathrm{pH}$ value of the solution higher than 6. Each wash process included $2500 \mathrm{rpm}$ for $3 \mathrm{~min}$ centrifugation and 2-4 min hand shaking. The as-prepared multilayer $\mathrm{Ti}_{3} \mathrm{C}_{2} \mathrm{~T}_{x}$ powder was dispersed in $100 \mathrm{~mL}$ deionized water by sonicating for $1 \mathrm{~h}$ and centrifuging at $3500 \mathrm{rpm}$ to obtain the $\mathrm{Ti}_{3} \mathrm{C}_{2} \mathrm{~T}_{x}$ colloidal solution. Finally, $\mathrm{Ti}_{3} \mathrm{C}_{2} \mathrm{~T}_{x}$ film was obtained by vacuum filtered on a cellulose membrane filter.

Based on this, the $\mathrm{Ti}_{3} \mathrm{C}_{2} \mathrm{~T}_{x}$ film was dispersed in deionized water to obtain a uniform suspension $\left(1 \mathrm{mg} \cdot \mathrm{mL}^{-1}\right)$. Then, $\mathrm{HAuCl}_{4}(1 \mathrm{wt} \%)$ solution with different volumes of 125,250 , and $500 \mu \mathrm{L}$ was slowly added to $30 \mathrm{~mL} \mathrm{Ti}_{3} \mathrm{C}_{2} \mathrm{~T}_{x}$ aqueous solution. The corresponding mixture was named as MXene/AuNPs-1 (2.4 wt $\%$ AuNPs), MXene/AuNPs-2 (4.8 wt\% AuNPs), and MXene/AuNPs-3 (9.6 wt $\%$ AuNPs), respectively. After stirring for $20 \mathrm{~min}$, the obtained composite 
suspension was filtered on a cellulose membrane filter by vacuum filtration, and dried at room temperature for $24 \mathrm{~h}$. Then, flexible and freestanding MXene/AuNPs composite film (about $30 \mathrm{mg}$ ) was obtained.

\section{3 Characterization}

$\mathrm{Ti}_{3} \mathrm{C}_{2} \mathrm{~T}_{x}$ and MXene/AuNPs samples were characterized by a scanning electron microscope (SEM, FEI, Nova 230, USA), with an energy dispersive spectrometer (EDS, Apollo XLT SDD, Japan), X-ray powder diffraction (XRD, TTRIII, Rigaku, Japan), a transmission electron microscope (TEM, FEI, Tecnai G2 F30, USA), and an X-ray photoelectron spectroscope (XPS, VG Multilab 2009, Al K $\alpha, \mathrm{UK})$. The specific surface area was determined from the nitrogen adsorption-desorption isotherm measured at $77 \mathrm{~K}$ using Brunauer-EmmettTeller model (Micromeritics ASAP 2010, BET, USA). The conductivity of samples was characterized by the precision source/measurement unit (Keysight B2911, USA).

\section{4 Electrochemical measurements}

CHI 660E electrochemical workstation was used in all electrochemical measurements. In a three-electrode system, electrochemical properties of $\mathrm{Ti}_{3} \mathrm{C}_{2} \mathrm{~T}_{x}$ and MXene/AuNPs electrode were studied by cyclic voltammetry (CV), galvanostatic charging/discharging (GCD), and electrochemical impedance spectroscopy (EIS) in $1 \mathrm{M} \mathrm{H}_{2} \mathrm{SO}_{4}$ electrolyte. $\mathrm{Ag} / \mathrm{AgCl}$ was served as reference electrodes, $\mathrm{Pt}$ sheet was used as the counter electrode, and $\mathrm{Ti}_{3} \mathrm{C}_{2} \mathrm{~T}_{x}$ and MXene/AuNPs were served as the working electrode. A symmetric supercapacitor was sandwiched in a coin cell with MXene/AuNPs composite film ( $\Phi 16 \mathrm{~mm}$ in diameter) as electrode, filter paper ( $\Phi 20 \mathrm{~mm}$ in diameter $)$ as separator, and $1 \mathrm{M} \mathrm{H}_{2} \mathrm{SO}_{4}$ as electrolyte.

\section{Results and discussion}

\section{1 Characterization and preparation mechanism}

Fabrication of MXene/AuNPs composite film is shown in Fig. 1. By selective etching of $\mathrm{Al}$ layer of $\mathrm{Ti}_{3} \mathrm{AlC}_{2}$ powders, multilayer $\mathrm{Ti}_{3} \mathrm{C}_{2} \mathrm{~T}_{x}$ powders are obtained. Then, multilayer $\mathrm{Ti}_{3} \mathrm{C}_{2} \mathrm{~T}_{x}$ powders are sonicated into $\mathrm{Ti}_{3} \mathrm{C}_{2} \mathrm{~T}_{x}$ nanosheets. There are functional groups on the surface of $\mathrm{Ti}_{3} \mathrm{C}_{2} \mathrm{~T}_{x}$ nanosheets, such as $-\mathrm{F},-\mathrm{O}$, and $-\mathrm{OH}$. These functional groups make $\mathrm{Ti}_{3} \mathrm{C}_{2} \mathrm{~T}_{x}$ nanosheets have a dispensability in aqueous solutions. Finally, adding the $\mathrm{HAuCl}_{4}$ solution into $\mathrm{Ti}_{3} \mathrm{C}_{2} \mathrm{~T}_{x}$ nanosheet dispersion, the composite material will be formed due to the reduction reaction between $\mathrm{AuCl}_{4}^{-}$and the functional groups on the surface of $\mathrm{Ti}_{3} \mathrm{C}_{2} \mathrm{~T}_{x}$ nanosheets. Additionally, this film obtained by vacuum filtration can be easily wrapped around a glass bar and retain its integrity, as showed in Fig. 1, indicating good flexibility.

From Fig. 2, the high diffraction peak at $6.9^{\circ}$ for $\mathrm{Ti}_{3} \mathrm{C}_{2} \mathrm{~T}_{x}$ corresponds to the (002) facet, suggesting the existing of the $\mathrm{Ti}_{3} \mathrm{C}_{2} \mathrm{~T}_{x}$ phase. For the MXene/AuNPs composite film, the (002) peak positions of $\mathrm{Ti}_{3} \mathrm{C}_{2} \mathrm{~T}_{x}$ do not vary, implying the presence of MXene and no $\mathrm{Au}$ embedded into the lattice of MXene [5]. The four diffraction peaks, as shown in Fig. 2, at $38.1^{\circ}, 44.4^{\circ}$, $64.5^{\circ}$, and $77.5^{\circ}$, correspond to (111), (200), (220), and (311) planes of AuNPs, respectively. As $\mathrm{HAuCl}_{4}$ content increasing, the four diffraction peaks become stronger and sharper, implying that the content of AuNPs decorated on $\mathrm{Ti}_{3} \mathrm{C}_{2} \mathrm{~T}_{x}$ is increasing.

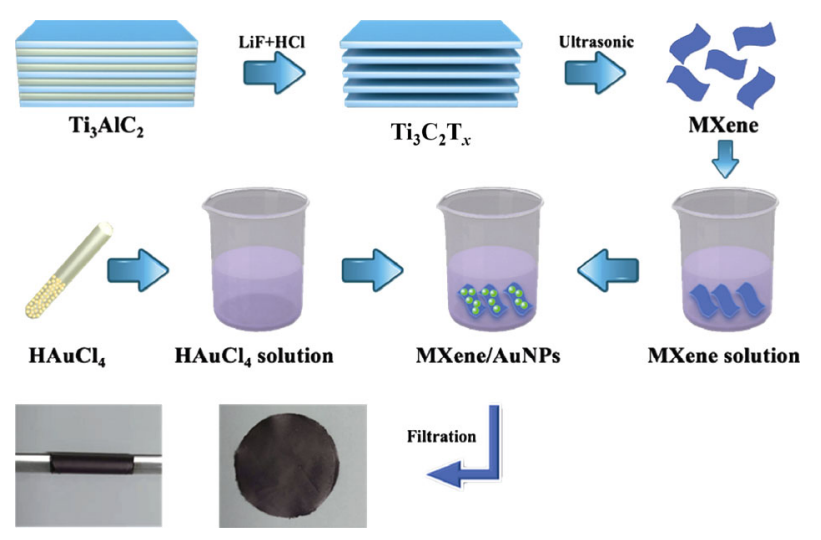

Fig. 1 Schematic of the synthesis of MXene/AuNPs composite film.

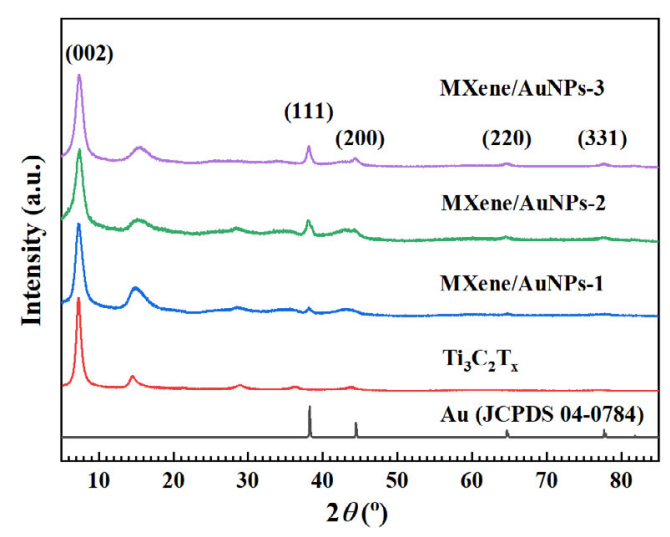

Fig. 2 XRD patterns of MXene/AuNPs, $\mathrm{Ti}_{3} \mathrm{C}_{2} \mathrm{~T}_{x}$, and AuNPs. 
In view of the morphology of the pure $\mathrm{Ti}_{3} \mathrm{C}_{2} \mathrm{~T}_{x}$ film (Fig. $\mathrm{S} 1$ in the Electronic Supplementary Material (ESM)), the surface is crumpled (Fig. S1(a) in the $\mathrm{ESM})$. The $\mathrm{Ti}_{3} \mathrm{C}_{2} \mathrm{~T}_{x}$ nanosheets have analogous wrinkles and high transparency, which are similar to graphene features (Fig. S1(b) in the ESM). The high resolution TEM (HRTEM) image shows that the fringe spacing of $0.253 \mathrm{~nm}$ corresponds to the (002) lattice plane of $\mathrm{Ti}_{3} \mathrm{C}_{2} \mathrm{~T}_{x}$ (Fig. $\mathrm{S} 1$ (c) in the ESM). The selected area electron diffraction (SAED) pattern (Fig. S1(d) in the ESM) shows characteristic diffraction rings corresponding to the (002) planes of $\mathrm{Ti}_{3} \mathrm{C}_{2} \mathrm{~T}_{x}$, clearly revealing the polycrystal of $\mathrm{Ti}_{3} \mathrm{C}_{2} \mathrm{~T}_{x}$. As shown in Figs. 3(a) and 3(b), MXene/AuNPs composite film exhibits similar morphologies of pure $\mathrm{Ti}_{3} \mathrm{C}_{2} \mathrm{~T}_{x}$ film, but the surface is relatively rougher. Figures $3(\mathrm{~b})$ and $3(\mathrm{~d})$ reveal a uniform distribution of AuNPs on $\mathrm{Ti}_{3} \mathrm{C}_{2} \mathrm{~T}_{x}$ nanosheets with the average particle size about $23 \mathrm{~nm}$ (Fig. 3(e)). The film exhibits crumpled morphology stacked by layers (Fig. 3(c)). And the intercalation of AuNPs has little effect to the thickness of MXene films. As shown in Fig. S2 in the ESM, the thickness of MXene films with different amounts of AuNPs at the same areal weight loading is almost $6.6 \mu \mathrm{m}$. As shown in Fig. 3(e), the HRTEM image shows that the fringe spacings of 0.235 and $0.203 \mathrm{~nm}$ correspond to the (111) and (200) lattice planes of $\mathrm{Au}$, respectively. The SAED pattern (Fig. 3(f)) shows characteristic diffraction rings corresponding to the (200) and (220) planes of Au. Figure S3 and Table S1 in the ESM show the EDS of MXene/AuNPs composite, indicating that AuNPs are decorated on the surface of $\mathrm{Ti}_{3} \mathrm{C}_{2} \mathrm{~T}_{x}$ nanosheets after $\mathrm{HAuCl}_{4}$ treatment in the absence of reducing agents and under the ambient condition. As shown in Fig. S4 in the ESM, the elemental mapping images indicate the even distribution of AuNPs.

The full XPS spectra of $\mathrm{Ti}_{3} \mathrm{C}_{2} \mathrm{~T}_{x}$ and MXene/AuNPs are shown in Fig. 4(a). The presence of MXene together with $-\mathrm{F},-\mathrm{Cl},-\mathrm{OH}$, and $-\mathrm{O}$ groups can be proved by observing the common peaks of Ti $2 \mathrm{p}, \mathrm{C} 1 \mathrm{~s}$, $\mathrm{F} 1 \mathrm{~s}, \mathrm{Cl} 2 \mathrm{p}$, and $\mathrm{O} 1 \mathrm{~s}$ from 0 to $800 \mathrm{eV}$. The $\mathrm{C} 1 \mathrm{~s}$ spectra of $\mathrm{Ti}_{3} \mathrm{C}_{2} \mathrm{~T}_{x}$ and MXene/AuNPs both have four characteristic peaks locating at 288.9, 286.4, 284.8, and $282.1 \mathrm{eV}$, as shown in Figs. 4(b) and 4(f), which correspond to the groups of $\mathrm{O}-\mathrm{C}=\mathrm{O}, \mathrm{C}-\mathrm{OH}, \mathrm{C}-\mathrm{C}$, and $\mathrm{C}-\mathrm{Ti}-\mathrm{O}$, respectively [22,23]. As shown in Figs. 4(c) and $4(\mathrm{~g})$, the Ti $2 \mathrm{p}$ spectra of $\mathrm{Ti}_{3} \mathrm{C}_{2} \mathrm{~T}_{x}$ and MXene/ AuNPs are both indexed with three characteristic peaks locating at 454.5, 455.9, and 457.4, corresponding to tetravalent $\mathrm{Ti}-\mathrm{C}$, $\mathrm{Ti}-\mathrm{X}\left(\mathrm{TiC}_{x}, x<1\right)$, and $\mathrm{Ti}_{x} \mathrm{O}_{y}$, but there is a characteristic peak locating at $458.8 \mathrm{eV}$ in the Ti $2 p$ spectra of MXene/AuNPs corresponding to $\mathrm{TiO}_{2}$, indicating the slight oxidation of $\mathrm{Ti}_{3} \mathrm{C}_{2} \mathrm{~T}_{x}$ after mixing with $\mathrm{HAuCl}_{4}$ [24-26]. The $\mathrm{O}$ 1s spectra of $\mathrm{Ti}_{3} \mathrm{C}_{2} \mathrm{~T}_{x}$ and MXene/AuNPs both have four characteristic peaks locating at 529.8, 531.2, 532.0, and $533.8 \mathrm{eV}$, corresponding to the groups of absorbed $\mathrm{O}, \mathrm{C}-\mathrm{Ti}-\mathrm{O}_{x}$, $\mathrm{C}-\mathrm{Ti}-\mathrm{OH}$, and $\mathrm{C}-\mathrm{OH}$, respectively, as shown in Figs. 4(d) and 4(h). The peak locating at $532.8 \mathrm{eV}$ corresponds to $\mathrm{OR}$, indicating the presence of organic compounds. The content of $\mathrm{O}$ of MXene/AuNPs reduces by $27 \%$ compared to that of $\mathrm{Ti}_{3} \mathrm{C}_{2} \mathrm{~T}_{x}$, as determined by XPS. As shown in Fig. 4(e), Au 4f spectrum of MXene/ AuNPs shows two characteristic peaks locating at 84.0 and $87.7 \mathrm{eV}$ where the binding energy difference is $3.7 \mathrm{eV}$, manifesting the presence of $\mathrm{Au}^{0}$.
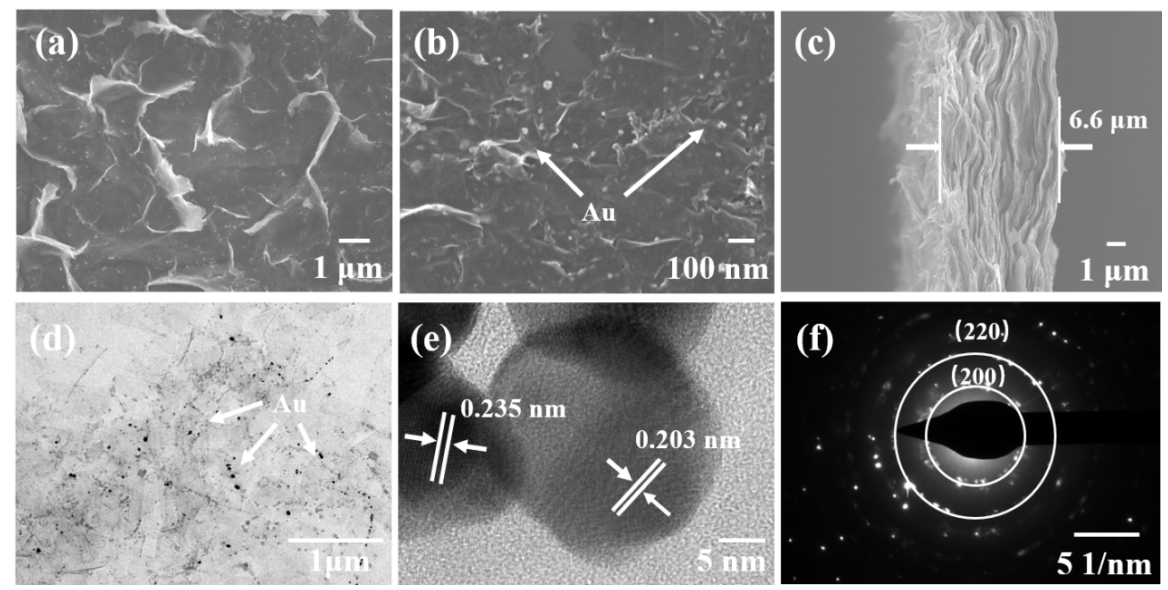

Fig. 3 (a, b) Different magnification SEM images of MXene/AuNPs composite film, (c) the cross-sectional SEM image of MXene/AuNPs composite film, (d) TEM image of MXene/AuNPs composite, (e) HRTEM image of MXene/AuNPs composite, and (f) SAED pattern of MXene/AuNPs composite. 

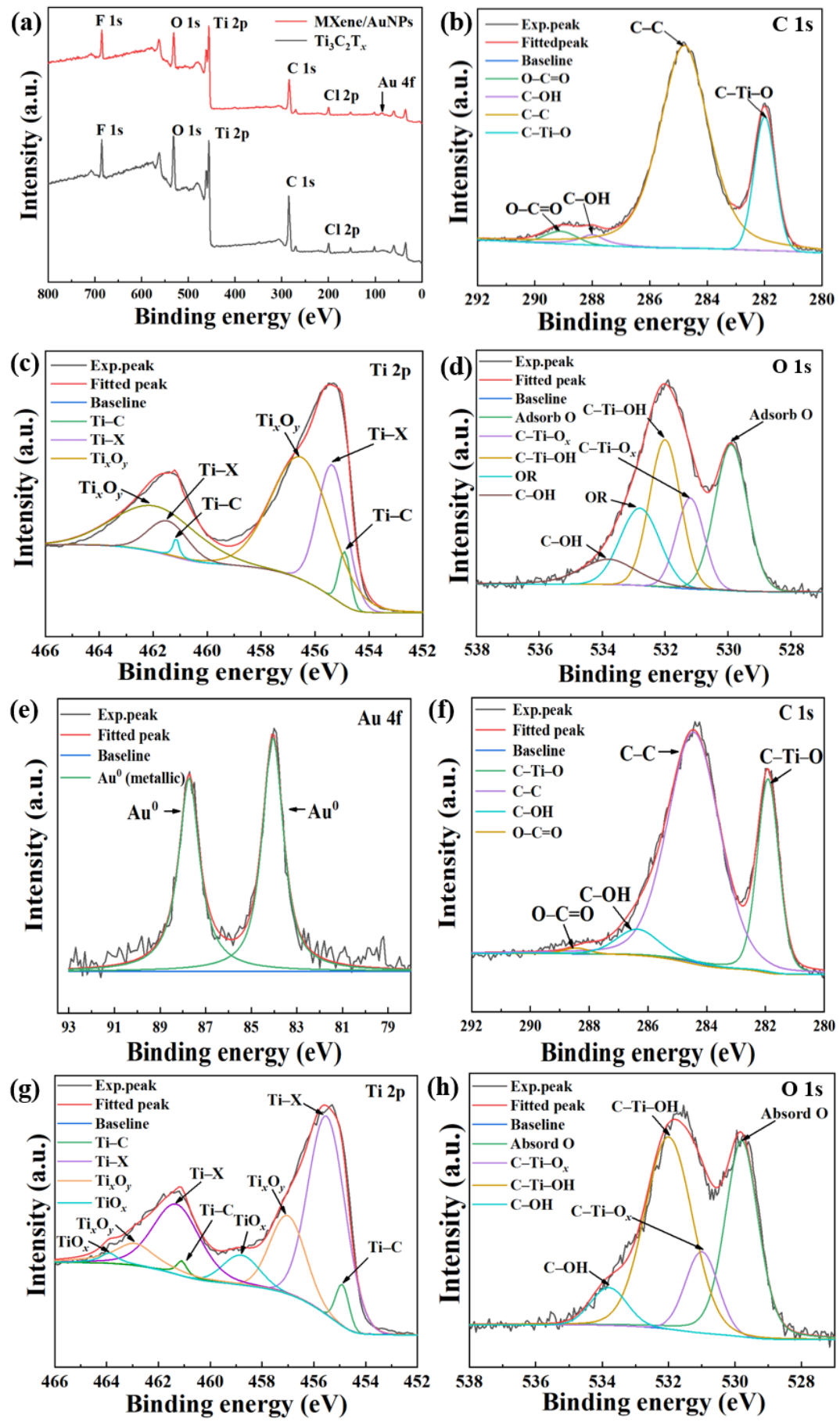

Fig. 4 (a) XPS full profile of $\mathrm{Ti}_{3} \mathrm{C}_{2} \mathrm{~T}_{x}$ and MXene/AuNPs samples. High resolution XPS spectra of (b) $\mathrm{C} 1 \mathrm{~s}$ for $\mathrm{Ti}_{3} \mathrm{C}_{2} \mathrm{~T}_{x},(\mathrm{c}) \mathrm{Ti}$ $2 \mathrm{p}$ for $\mathrm{Ti}_{3} \mathrm{C}_{2} \mathrm{~T}_{x}$, (d) O $1 \mathrm{~s}$ for $\mathrm{Ti}_{3} \mathrm{C}_{2} \mathrm{~T}_{x}$, (e)Au $4 \mathrm{f}$ for MXene/AuNPs, (f) $\mathrm{C} 1 \mathrm{~s}$ for MXene/AuNPs, (g) Ti 2p for MXene/AuNPs, and (h) O $1 \mathrm{~s}$ for MXene/AuNPs.

From above results, the AuNPs are assembled and decorated on the surfaces of $\mathrm{Ti}_{3} \mathrm{C}_{2} \mathrm{~T}_{x}$ nanosheets evenly. It should be pointed out that no additional reducing agent was added in this work. Cheng et al. [27] have demonstrated that MXenes with $-\mathrm{OH}$ terminations can reduce noble metal ions into zero-valent metals. Since the surfaces of $\mathrm{Ti}_{3} \mathrm{C}_{2} \mathrm{~T}_{x}$ nanosheets are covered by $-\mathrm{F}$,
$-\mathrm{O}$, and $-\mathrm{OH}, \mathrm{AuCl}_{4}^{-}$can be directly reduced by $-\mathrm{OH}$ and formed AuNPs [28].

$$
\mathrm{AuCl}_{4}^{-}+3 \mathrm{e} \rightarrow \mathrm{Au}^{0} \downarrow+4 \mathrm{Cl}^{-}
$$

which is consistent with reduction in $\mathrm{O}$ content calculated above.

The AuNPs and $\mathrm{Ti}_{3} \mathrm{C}_{2} \mathrm{~T}_{x}$ nanosheets are combined 
into a structure of conducting network, which depicts the pronounced contributions of AuNPs to the enhancement of conductivity. This structure contributes to the electrolyte penetration, the ion transport, and the active site increase in electrochemical reaction.

\section{2 Electrochemical property of MXene/AuNPs electrode}

Figure 5(a) shows the $\mathrm{CV}$ curves of $\mathrm{Ti}_{3} \mathrm{C}_{2} \mathrm{~T}_{x}$ and MXene/AuNPs electrodes at $5 \mathrm{mV} \cdot \mathrm{s}^{-1}$ in $1 \mathrm{M} \mathrm{H}_{2} \mathrm{SO}_{4}$ solution. Due to the contribution of pseudocapacitance derived from the varied valence of transition metal $\mathrm{Ti}$ atoms, $\mathrm{CV}$ curves of $\mathrm{Ti}_{3} \mathrm{C}_{2} \mathrm{~T}_{x}$ and MXene/AuNPs film exhibit deformed rectangle shape. According to the $\mathrm{CV}$ curves of $\mathrm{Ti}_{3} \mathrm{C}_{2} \mathrm{~T}_{x}$, MXene/AuNPs-1, MXene/AuNPs-2, and MXene/AuNPs-3 electrodes, the specific capacitance is calculated as $228,234,278$, and $250 \mathrm{~F} \cdot \mathrm{g}^{-1}$, respectively. MXene/AuNP-2 composite electrode shows the largest $\mathrm{CV}$ area, which is about 1.2 times larger than that of $\mathrm{Ti}_{3} \mathrm{C}_{2} \mathrm{~T}_{x}$ electrode, indicating it possesses better electrochemical capacitive performance by wedging AuNPs. Figure 5(b) presents CV curves of MXene/AuNPs-2 varying from 5 to $100 \mathrm{mV} \cdot \mathrm{s}^{-1}$. The shape deformation of $\mathrm{CV}$ curves becomes heavier with the scan rate increasing. This phenomenon is possibly caused by slow ion response at high rates. GCD measurement was performed to investigate electrochemical performance of MXene/AuNPs-2 at current density ranging from 1 to $10 \mathrm{~A} \cdot \mathrm{g}^{-1}$ as shown in Fig. 5(c). GCD curves of MXene/AuNPs are almost symmetric and linear triangles, indicating that MXene/AuNPs has reversible charge/discharge process and good capacitance behavior. The inset of Fig. 5(c) is the coulombic efficiency (CE) calculated from different current densities. The calculated results of $1,2,5$, and $10 \mathrm{~A} \cdot \mathrm{g}^{-1}$ are $72.8 \%, 86.5 \%, 97.4 \%$, and $99.5 \%$, respectively, showing that MXene/AuNPs has a high coulombic efficiency. In view of the rate capability as shown in Fig. 5(d), the specific capacitance of 5, 10, 20, 50, and $100 \mathrm{mV} / \mathrm{s}$ is $278,251,225,198$, and $181 \mathrm{~F} \cdot \mathrm{g}^{-1}$, respectively, indicating that MXene/AuNPs-2 retains about $65 \%$ of the initial capacitance. The good rate capability is attributed to the 2D structure and good conductivity of MXene/AuNPs, which allows rapid ion transport. It should be noted that the AuNPs have no capacitive contribution. Therefore, the specific
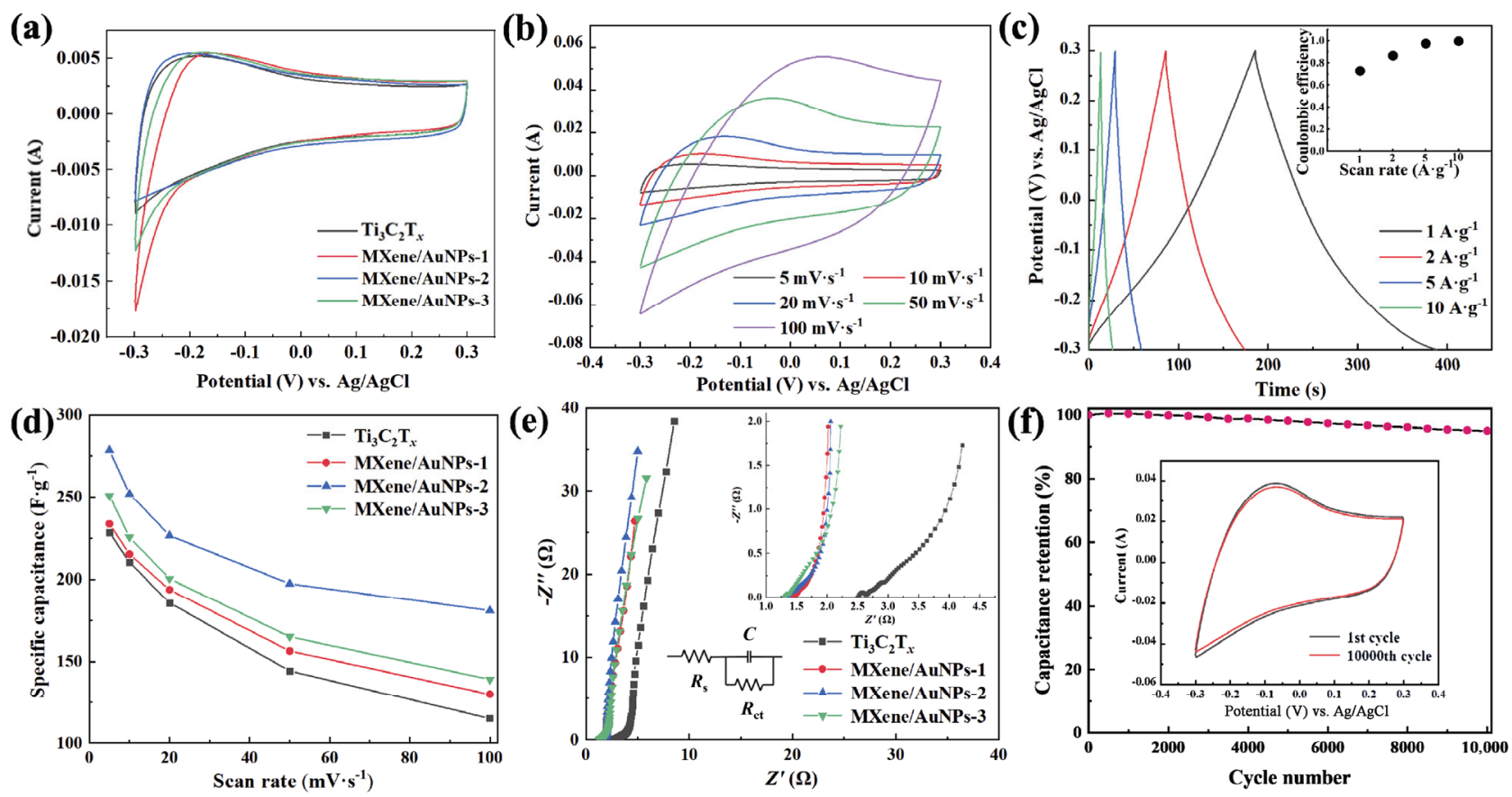

Fig. 5 (a) CV curves of $\mathrm{Ti}_{3} \mathrm{C}_{2} \mathrm{~T}_{x}$ and MXene/AuNPs electrodes; (b) CV curves of MXene/AuNPs-2 electrode with different scan rates; (c) GCDs of MXene/AuNPs-2 electrode with different current density, the inset is the CE calculated from different current density; (d) the specific capacitance of $\mathrm{Ti}_{3} \mathrm{C}_{2} \mathrm{~T}_{x}$ and MXene/AuNPs electrode vs. the scan rate; (e) Nyquist plots of $\mathrm{Ti}_{3} \mathrm{C}_{2} \mathrm{~T}_{x}$ and MXene/AuNPs electrode, the insets are the magnified high-frequency region and the equivalent circuit diagram of the electrochemical system; and (f) cycling performance of MXene/AuNPs-2 after 10,000 cycles. 
capacitance decreases with the excessive addition of $\mathrm{HAuCl}_{4}$ solution. The Nyquist plots shown in Fig. 5(e) are composed of two regions with a semicircle at the high-frequency part and an inclined line at the low-frequency part. The intercept of the $x$-axis represents the equivalent series resistance, which is related to the resistance of the electrode $[29,30]$. The equivalent series resistance of the MXene/AuNPs-2 (1.4 $\Omega$ ) shows the lowest resistance value compared to those of the $\mathrm{Ti}_{3} \mathrm{C}_{2} \mathrm{~T}_{x}(2.5 \Omega)$, MXene/AuNPs-1 $(1.5 \Omega)$, and MXene/AuNPs-3 (1.4 $\Omega$ ) electrodes. The semicircle represents the charge transfer resistance $\left(R_{\mathrm{ct}}\right)$ at the electrode-electrolyte interface, which is $0.008,0.007$, 0.006 , and $0.008 \Omega$ for $\mathrm{Ti}_{3} \mathrm{C}_{2} \mathrm{~T}_{x}$, MXene/AuNPs-1, MXene/AuNPs-2, and MXene/AuNPs-3, respectively. The Warburg impedance stemming from the slope of the curve reflects the ion diffusion of the electrode [31], which is $8.7,8.5,11.1$, and $8.3 \mathrm{~S}^{1 / 2}$ for $\mathrm{Ti}_{3} \mathrm{C}_{2} \mathrm{~T}_{x}$, MXene/AuNPs-1, MXene/AuNPs-2, and MXene/ AuNPs-3, respectively. Among them, the MXene/ AuNPs-2 electrode has the smallest $R_{\mathrm{ct}}$ and the highest Warburg impedance, indicating excellent ion diffusion capabilities. The mechanism of MXene/AuNPs-2 with the best electrochemical performance can be mainly attributed to the following points: i) Compared with MXene/AuNPs-1 (AuNPs of $\sim 18 \mathrm{~nm}$ in diameter and 0.43 at $\%$ ), MXene/AuNPs-2 (AuNPs of $\sim 23 \mathrm{~nm}$ in diameter and 0.95 at $\%$ ) with larger spacing of MXene nanosheets possesses higher conductivity for electronic, better diffusion for electrolyte and ions, and larger specific surface area for active sites (Fig. S5 and Table S1 in the ESM); ii) compared with MXene/AuNPs-3, as the increasing of content of $\mathrm{Au}(1.74 \mathrm{at} \%)$, AuNPs will aggregate and grow up $(\sim 50 \mathrm{~nm})$, which making the spacing of MXene nanosheets too large. This is harmful to the conductivity of MXene film (Fig. S5(d) in the ESM) [32]. Therefore, compared with MXene/AuNPs-1 and MXene/AuNPs-3, MXene/AuNPs-2 has the best electrochemical performance. The cyclic stability of MXene/AuNPs-2 electrodes is shown in Fig. 5(f). Notably, at $50 \mathrm{mV} \cdot \mathrm{s}^{-1}$, it exhibits excellent stability and the specific capacitance of MXene/AuNPs-2 still remains $95.0 \%$ after 10,000 cycles.

The high stability and good electrochemical performance of MXene/AuNPs composite electrode could be mainly derived from three aspects: (1) The intrinsic properties of MXene and gold. MXene possesses both excellent EDLC and pseudo-capacitance. Gold has excellent stability and conductivity. (2) As shown in Fig. 6(a), the AuNPs as the spacer prevents selfstacking between $\mathrm{Ti}_{3} \mathrm{C}_{2} \mathrm{~T}_{x}$ nanosheets and lead to more space between the tightly stacked nanosheets, which significantly enlarge specific surface area of the composite material [33]. The BET results of $\mathrm{Ti}_{3} \mathrm{C}_{2} \mathrm{~T}_{x}$ and MXene/AuNPs, as shown Fig. 6(b), reveal that the specific surface area of MXene/AuNPs is about 1.8 times larger than that of $\mathrm{Ti}_{3} \mathrm{C}_{2} \mathrm{~T}_{x}$. The larger specific surface area provides more active sites, improving the electrochemical performance. (3) The AuNPs as the spacer prevents self-stacking of $\mathrm{Ti}_{3} \mathrm{C}_{2} \mathrm{~T}_{x}$ nanosheets, which is beneficial for electrolyte penetration and the transport of ions [11]. As shown in Fig. 5(e), the smaller solution resistance $\left(R_{\mathrm{S}}\right)$ of MXene/AuNPs implies MXene/ AuNPs have faster ion response and lower inherent resistance. (4) The conductivity of MXene/AuNPs composite is significantly improved due to AuNPs' excellent conductivity and the structure of conducting network between the AuNPs and $\mathrm{Ti}_{3} \mathrm{C}_{2} \mathrm{~T}_{x}$ nanosheets [33]. As shown in Fig. S6 in the ESM, the conductivity of MXene/AuNPs is 1.67 times higher than that of $\mathrm{Ti}_{3} \mathrm{C}_{2} \mathrm{~T}_{x}$. (a)

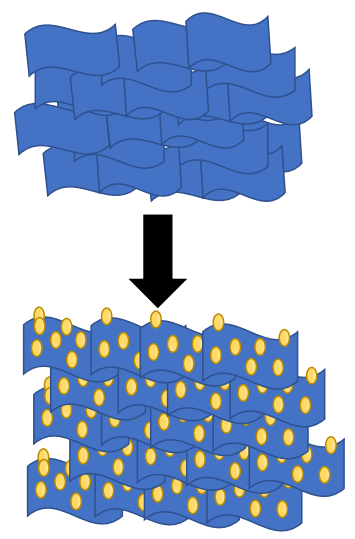

(b)

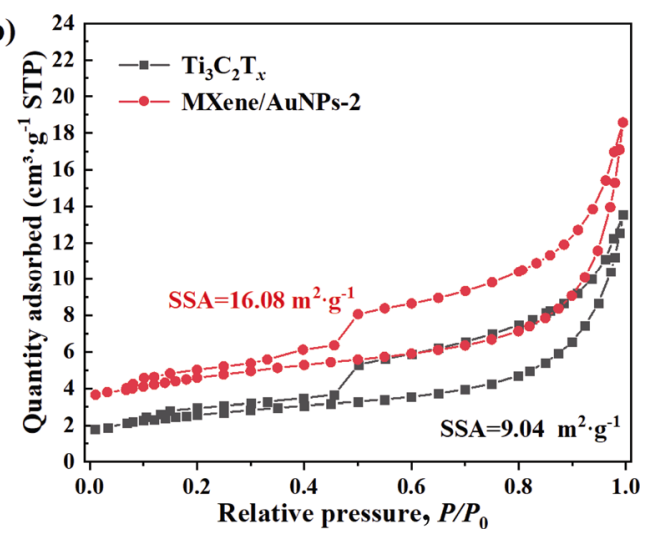

Fig. 6 (a) Schematic illustration of $\mathrm{Ti}_{3} \mathrm{C}_{2} \mathrm{~T}_{x}$ film and MXene/AuNPs film and (b) $\mathrm{N}_{2}$ adsorption-desorption curves of $\mathrm{Ti}_{3} \mathrm{C}_{2} \mathrm{~T}_{x}$ film and MXene/AuNPs film. 
Compared with the performances of the various MXene-based supercapacitors in Table 1, the specific capacitance of MXene/AuNPs with an electrolyte of $1 \mathrm{M} \mathrm{H}_{2} \mathrm{SO}_{4}$ in this work is calculated to be $278 \mathrm{~F} \cdot \mathrm{g}^{-1}$ $\left(5 \mathrm{mV} \cdot \mathrm{s}^{-1}\right)$. This value is 1.2 times higher than that of MXene (i.e., $238 \mathrm{~F} \cdot \mathrm{g}^{-1}$ ) and is better than that of $\mathrm{Ti}_{3} \mathrm{C}_{2} \mathrm{~T}_{x}-\mathrm{Cl}$ [34], $\mathrm{Ti}_{3} \mathrm{C}_{2} \mathrm{~T}_{x}$ clay [35], and $\mathrm{Ti}_{3} \mathrm{C}_{2} \mathrm{~T}_{x}-\mathrm{N}_{2} \mathrm{H}_{4}$ [36].

Table 1 Key parameters of various MXene-based supercapacitors

\begin{tabular}{|c|c|c|c|}
\hline Material & Electrolyte & Specific capacitance & Ref. \\
\hline $\mathrm{Ti}_{3} \mathrm{C}_{2} \mathrm{~T}_{x}-\mathrm{Cl}$ & $1 \mathrm{M} \mathrm{H}_{2} \mathrm{SO}_{4}$ & $180 \mathrm{~F} \cdot \mathrm{g}^{-1}\left(2 \mathrm{mV} \cdot \mathrm{s}^{-1}\right)$ & [34] \\
\hline $\mathrm{Ti}_{3} \mathrm{C}_{2} \mathrm{~T}_{x}$ clay & $1 \mathrm{M} \mathrm{H}_{2} \mathrm{SO}_{4}$ & $245 \mathrm{~F} \cdot \mathrm{g}^{-1}\left(2 \mathrm{mV} \cdot \mathrm{s}^{-1}\right)$ & [35] \\
\hline $\mathrm{Ti}_{3} \mathrm{C}_{2} \mathrm{~T}_{x}-\mathrm{N}_{2} \mathrm{H}_{4}$ & $1 \mathrm{M} \mathrm{H}_{2} \mathrm{SO}_{4}$ & $250 \mathrm{~F} \cdot \mathrm{g}^{-1}\left(5 \mathrm{mV} \cdot \mathrm{s}^{-1}\right)$ & {$[36]$} \\
\hline $\mathrm{Ti}_{3} \mathrm{C}_{2} \mathrm{~T}_{x}$ & $1 \mathrm{M} \mathrm{H}_{2} \mathrm{SO}_{4}$ & $238 \mathrm{~F} \cdot \mathrm{g}^{-1}\left(5 \mathrm{mV} \cdot \mathrm{s}^{-1}\right)$ & [37] \\
\hline $\mathrm{N}-\mathrm{Ti}_{3} \mathrm{C}_{2} \mathrm{~T}_{x}$ & $1 \mathrm{M} \mathrm{H}_{2} \mathrm{SO}_{4}$ & $192 \mathrm{~F} \cdot \mathrm{g}^{-1}\left(5 \mathrm{mV} \cdot \mathrm{s}^{-1}\right)$ & [38] \\
\hline $\mathrm{Ti}_{3} \mathrm{C}_{2} \mathrm{~T}_{x} / \mathrm{CNT}$ & $1 \mathrm{M} \mathrm{MgSO}_{4}$ & $117 \mathrm{~F} \cdot \mathrm{g}^{-1}\left(2 \mathrm{mV} \cdot \mathrm{s}^{-1}\right)$ & {$[39]$} \\
\hline PANI- $\mathrm{Ti}_{3} \mathrm{C}_{2} \mathrm{~T}_{x}$ & $1 \mathrm{M} \mathrm{Na}_{2} \mathrm{SO}_{4}$ & $164 \mathrm{~F} \cdot \mathrm{g}^{-1}\left(2 \mathrm{mV} \cdot \mathrm{s}^{-1}\right)$ & {$[40]$} \\
\hline $\mathrm{d}-\mathrm{Ti}_{3} \mathrm{C}_{2} \mathrm{~T}_{x}$ film & $1 \mathrm{M} \mathrm{KOH}$ & $140 \mathrm{~F} \cdot \mathrm{g}^{-1}\left(5 \mathrm{mV} \cdot \mathrm{s}^{-1}\right)$ & {$[41]$} \\
\hline $\mathrm{Ti}_{3} \mathrm{C}_{2} \mathrm{~T}_{x} / \mathrm{RGO}$ & $1 \mathrm{M} \mathrm{H}_{2} \mathrm{SO}_{4}$ & $140 \mathrm{~F} \cdot \mathrm{g}^{-1}\left(5 \mathrm{mV} \cdot \mathrm{s}^{-1}\right)$ & {$[42]$} \\
\hline $\mathrm{MnO}_{2} @ \mathrm{Ti}_{3} \mathrm{C}_{2} \mathrm{~T}_{x} / \mathrm{CNT}$ & $1 \mathrm{M} \mathrm{Na}_{2} \mathrm{SO}_{4}$ & $181.8 \mathrm{~F} \cdot \mathrm{g}^{-1}\left(5 \mathrm{mV} \cdot \mathrm{s}^{-1}\right)$ & {$[43]$} \\
\hline $\mathrm{Ti}_{3} \mathrm{C}_{2} \mathrm{~T}_{x} / \mathrm{AuNPs}$ & $1 \mathrm{M} \mathrm{H}_{2} \mathrm{SO}_{4}$ & $278 \mathrm{~F} \cdot \mathrm{g}^{-1}\left(5 \mathrm{mV} \cdot \mathrm{s}^{-1}\right)$ & This work \\
\hline
\end{tabular}

\section{3 Electrochemical performance of MXene/AuNPs symmetric supercapacitor}

To evaluate the performance of MXene/AuNPs-2 as supercapacitor, the $\mathrm{CV}, \mathrm{GCD}$, and EIS were studied. In the working potential window of $0.0-0.6 \mathrm{~V}$ with the scan rates ranging from 5 to $100 \mathrm{mV} \cdot \mathrm{s}^{-1}$, as shown in Fig. 7(a), CV curves are almost rectangular, demonstrating its ideal characteristic of electrical double-layer capacitor and excellent rate capability. At current density ranging from 1 to $10 \mathrm{~A} \cdot \mathrm{g}^{-1}$, the GCD curves demonstrate a good capacitive performance due to its good linear potential-time profiles as well as nearly symmetrical with their discharging counterpart (Fig. 7(b)). There is no obvious semicircular in the high-frequency Nyquist diagram (Fig. 7(c)), indicating the low $R_{\mathrm{ct}}$ and the good electrical conductivity of the composite. The cyclic stability of MXene/AuNPs-2 electrodes is shown in Fig. 7(d). The specific capacitance of symmetric supercapacitor remains $95.0 \%$ after 10,000 cycles at $50 \mathrm{mV} \cdot \mathrm{s}^{-1}$, which exhibits excellent stability. As shown in Fig. S3 in the ESM with the increase of the scanning rate, the gravimetric capacitance obviously decreases, which is due to slow ion transport rate. By calculation, the gravimetric capacitance is 213.8 ,
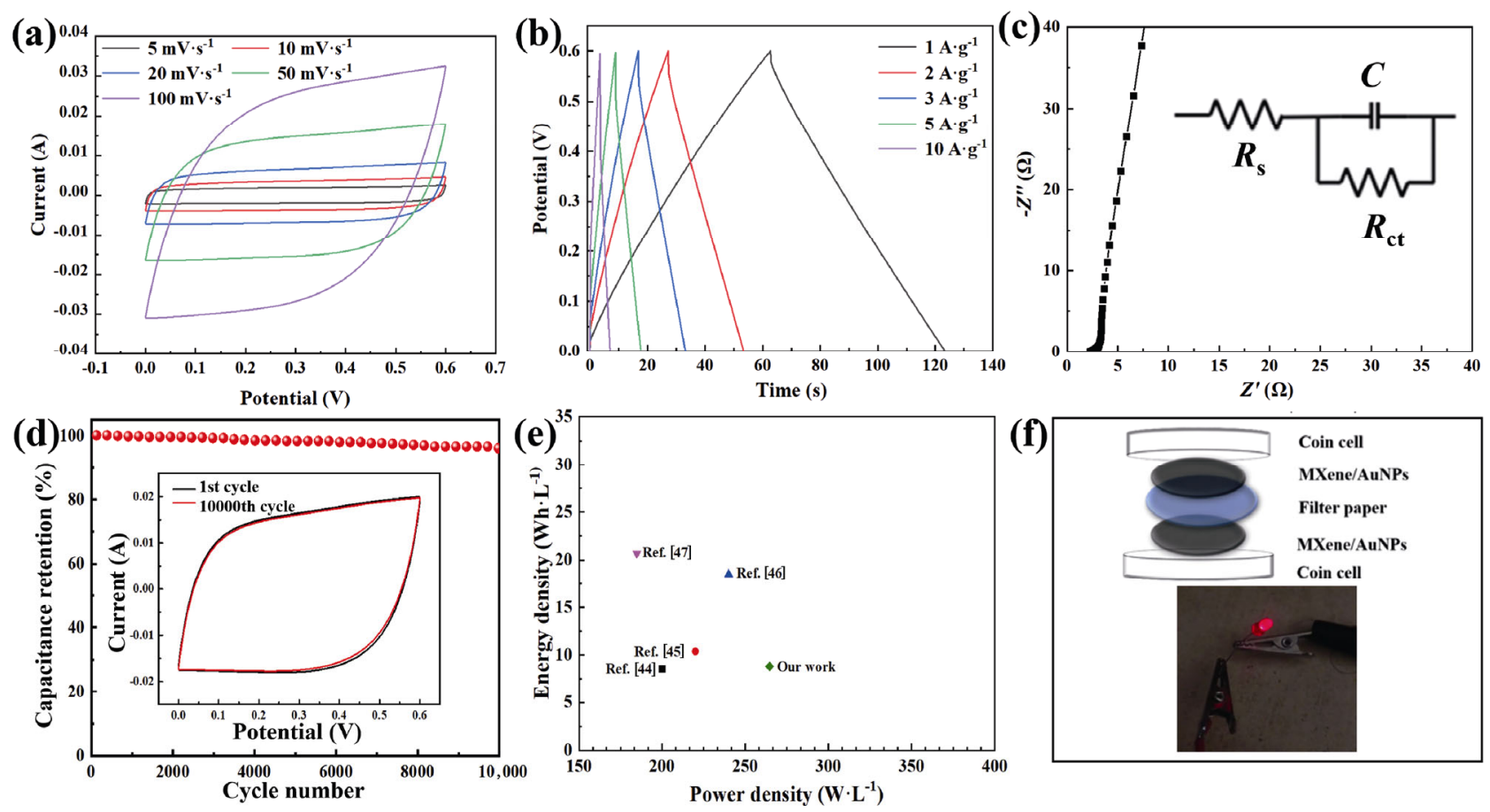

Fig. 7 (a) CV curves of MXene/AuNPs-2 symmetric supercapacitor; (b) GCDs of the supercapacitor; (c) Nyquist plots of the supercapacitor, the inset is the equivalent circuit diagram of the electrochemical system; (d) cycling performance of MXene/AuNPs-2 symmetric supercapacitor after 10,000 cycles; (e) volumetric energy and power density for the supercapacitors compared with those in other literature; and (f) schematic diagram of structure of symmetric supercapacitor. 
$196.1,186.4,160.9$, and $141.1 \mathrm{~F} \cdot \mathrm{g}^{-1}$ from 100 to $5 \mathrm{mV} \cdot \mathrm{s}^{-1}$, which can be also found in Fig. S7 in the ESM. Figure 7(e) shows the comparation of the volumetric energy density and power density of MXene/AuNPs with other supercapacitors reported in the literature [44-47]. Calculated from the data in Fig. 7(a), the volumetric power density and energy density at $5,10,20,50$, and $100 \mathrm{mV} \cdot \mathrm{s}^{-1}$ are calculated to be $264.6,485.3,922.7,1991.1$, and $3492.2 \mathrm{~W} \cdot \mathrm{L}^{-1}$, and $8.82,8.09,7.69,6.64$, and $5.82 \mathrm{Wh} \cdot \mathrm{L}^{-1}$, respectively. Compared with MXene-based supercapacitor reported in the literature, i.e., $\mathrm{rGO} / / \mathrm{MXene}\left(8.6 \mathrm{Wh} \cdot \mathrm{L}^{-1}, 200 \mathrm{~W} \cdot \mathrm{L}^{-1}\right)$ [44], Ni-dMXNC//MXene $\left(10.4 \mathrm{Wh} \cdot \mathrm{L}^{-1}, 220 \mathrm{~W} \cdot \mathrm{L}^{-1}\right)$ [45], MP-MX $1.5\left(20.7 \mathrm{Wh} \cdot \mathrm{L}^{-1}, 184.8 \mathrm{~W} \cdot \mathrm{L}^{-1}\right)$ [46], and $\mathrm{Ti}_{3} \mathrm{C}_{2} \mathrm{~T}_{x}$ paper $\left(18.5 \mathrm{Wh} \cdot \mathrm{L}^{-1}, 240 \mathrm{~W} \cdot \mathrm{L}^{-1}\right)$ [47], the energy density of MXene/AuNPs in this work is comparable to these MXene-based symmetric or asymmetric supercapacitor devices while the power density is higher than those. (Table S2 in the ESM). As shown in Fig. S8 in the ESM, the time constant is calculated as $5 \mathrm{~s}$. Leakage current of the sueprcapacitor has been recorded at $0.6 \mathrm{~V}$. Leakage current diminishes significantly to $0.049 \mathrm{~mA}$ within few seconds and then it further reduces to its low value of $0.023 \mathrm{~mA}$. We performed self-discharge test of the sueprcapacitor for $2 \mathrm{~h}$. Figure S9 in the ESM displays the good capability of $0.32 \mathrm{~V}$ after $2 \mathrm{~h}$ self-discharge test. In addition, a red LED $(1 \mathrm{~W}, 2.6-2.8 \mathrm{~V}, 350 \mathrm{~mA})$ is successfully powered by three prepared symmetric supercapacitors in series, as shown in Fig. 7(f), convincingly demonstrating the energy storage capacity of MXene/AuNPs symmetric supercapacitor.

\section{Conclusions}

In summary, MXene/AuNPs composite is fabricated with AuNPs evenly distributed on the surface of $\mathrm{Ti}_{3} \mathrm{C}_{2} \mathrm{~T}_{x}$ nanosheets. The obtained AuNPs are directly reduced from $\mathrm{AuCl}_{4}^{-}$by the groups of $-\mathrm{OH}$ on the surface of $\mathrm{Ti}_{3} \mathrm{C}_{2} \mathrm{~T}_{x}$ nanosheets. The AuNPs and $\mathrm{Ti}_{3} \mathrm{C}_{2} \mathrm{~T}_{x}$ nanosheets are combined into a structure of conducting network, which contributes to rapid electron transfer in electrochemical reactions. Composite electrodes (MXene decorated on $4.8 \mathrm{wt} \%$ AuNPs) show enhanced charge storage ability with a capacitance of $278 \mathrm{~F} \cdot \mathrm{g}^{-1}$ at $5 \mathrm{mV} \cdot \mathrm{s}^{-1}$. The cyclic stability reaches $95.0 \%$ after 10,000 cycles. Furthermore, a MXene/AuNPs symmetric supercapacitor with filter paper as separator and $\mathrm{H}_{2} \mathrm{SO}_{4}$ as electrolyte, exhibits a high volumetric energy density of $8.82 \mathrm{Wh} \cdot \mathrm{L}^{-1}$ at a power density of $264.6 \mathrm{~W} \cdot \mathrm{L}^{-1}$. The integrated device can power a red LED demonstrating its energy storage capacity.

\section{Acknowledgements}

This work is supported by the National Science Fund for Distinguished Young Scholars (No. 52025041), the National Natural Science Foundation of China (Nos. 51974021, 51902020, and 51904021), the Fundamental Research Funds for the Central Universities (Nos. FRF-TP-18-045A1 and FRF-TP-19-004B2Z), the National Postdoctoral Program for Innovative Talents (No. BX20180034), China Postdoctoral Science Foundation (No. 2018M641192), and the Open Foundation of Guangxi Key Laboratory of Processing for Non-ferrous Metals and Featured Materials, Guangxi University (No. 2021GXYSOF12).

\section{Electronic Supplementary Material}

Supplementary material is available in the online version of this article at https://doi.org/10.1007/s40145-021-0491-0.

\section{References}

[1] Wang BX, Zhou AG, Liu FF, et al. Carbon dioxide adsorption of two-dimensional carbide MXenes. $J A d v$ Ceram 2018, 7: 237-245.

[2] Yang CH, Tang Y, Tian YP, et al. Flexible nitrogen-doped 2D titanium carbides (MXene) films constructed by an $e x$ situ solvothermal method with extraordinary volumetric capacitance. Adv Energy Mater 2018, 8: 1802087.

[3] Gao YP, Wang LB, Li ZY, et al. Electrochemical performance of $\mathrm{Ti}_{3} \mathrm{C}_{2}$ supercapacitors in $\mathrm{KOH}$ electrolyte. $J$ Adv Ceram 2015, 4: 130-134.

[4] Tan CL, Cao XH, Wu XJ, et al. Recent advances in ultrathin two-dimensional nanomaterials. Chem Rev 2017, 117: 6225-6331.

[5] Naguib M, Mochalin VN, Barsoum MW, et al. 25th anniversary article: MXenes new family of twodimensional materials. Adv Mater 2014, 26: 992-1005.

[6] Liang X, Garsuch A, Nazar LF. Sulfur cathodes based on conductive MXene nanosheets for high-performance lithium-sulfur batteries. Angewandte Chemie Int Ed 2015, 54: 3907-3911.

[7] Berdiyorov GR, Madjet ME, Mahmoud KA. Ionic sieving through $\mathrm{Ti}_{3} \mathrm{C}_{2}(\mathrm{OH})_{2}$ MXene: First-principles calculations. Appl Phys Lett 2016, 108: 113110.

[8] Naguib M, Kurtoglu M, Presser V, et al. Two-dimensional 
nanocrystals produced by exfoliation of $\mathrm{Ti}_{3} \mathrm{AlC}_{2} . A d v$ Mater 2011, 23: 4248-4253.

[9] Liu YT, Zhu XD, Pan L. Hybrid architectures based on 2D MXenes and low-dimensional inorganic nanostructures: Methods, synergies, and energy-related applications. Small 2018, 14: 1803632.

[10] Xu YX, Lin ZY, Huang XQ, et al. Functionalized graphene hydrogel-based high-performance supercapacitors. $A d v$ Mater 2013, 25: 5779-5784.

[11] Yu HT, Xu PC, Lee DW, et al. Porous-layered stack of functionalized AuNP-rGO (gold nanoparticles-reduced graphene oxide) nanosheets as a sensing material for the micro-gravimetric detection of chemical vapor. $J$ Mater Chem A 2013, 1: 4444.

[12] Zhao MQ, Ren CE, Ling Z, et al. Flexible MXene/carbon nanotube composite paper with high volumetric capacitance. Adv Mater 2015, 27: 339-345.

[13] Yan J, Ren CE, Maleski K, et al. Flexible MXene/graphene films for ultrafast supercapacitors with outstanding volumetric capacitance. Adv Funct Mater 2017, 27: 1701264.

[14] Xu SK, Wei GD, Li JZ, et al. Flexible MXene-graphene electrodes with high volumetric capacitance for integrated co-cathode energy conversion/storage devices. J Mater Chem A 2017, 5: 17442-17451.

[15] Boota M, Anasori B, Voigt C, et al. Pseudocapacitive electrodes produced by oxidant-free polymerization of pyrrole between the layers of 2D titanium carbide (MXene). Adv Mater 2016, 28: 1517-1522.

[16] Chen C, Boota M, Xie XQ, et al. Charge transfer induced polymerization of EDOT confined between 2D titanium carbide layers. J Mater Chem A 2017, 5: 5260-5265.

[17] VahidMohammadi A, Moncada J, Chen HZ, et al. Thick and freestanding MXene/PANI pseudocapacitive electrodes with ultrahigh specific capacitance. J Mater Chem A 2018, 6: 22123-22133.

[18] Khamlich S, Khamliche T, Dhlamini MS, et al. Rapid microwave-assisted growth of silver nanoparticles on 3D graphene networks for supercapacitor application. $J$ Colloid Interface Sci 2017, 493: 130-137.

[19] Dong XC, Huang W, Chen P. In situ synthesis of reduced graphene oxide and gold nanocomposites for nanoelectronics and biosensing. Nanoscale Res Lett 2010, 6: 1-6.

[20] Yan Y, Wang TY, Li XR, et al. Noble metal-based materials in high-performance supercapacitors. Inorg Chem Front 2017, 4: 33-51.

[21] Alhabeb M, Maleski K, Anasori B, et al. Guidelines for synthesis and processing of two-dimensional titanium carbide $\left(\mathrm{Ti}_{3} \mathrm{C}_{2} \mathrm{~T}_{x}\right.$ MXene). Chem Mater 2017, 29: 76337644.

[22] Zhang LB, Chen GY, Hedhili MN, et al. Three-dimensional assemblies of graphene prepared by a novel chemical reduction-induced self-assembly method. Nanoscale 2012, 4: 7038 .

[23] Tang Y, Zhu JF, Yang CH, et al. Enhanced capacitive performance based on diverse layered structure of two-dimensional $\mathrm{Ti}_{3} \mathrm{C}_{2}$ MXene with long etching time. $J$ Electrochem Soc 2016, 163: A1975-A1982.

[24] Rakhi RB, Ahmed B, Hedhili MN, et al. Effect of postetch annealing gas composition on the structural and electrochemical properties of $\mathrm{Ti}_{2} \mathrm{CT}_{x}$ MXene electrodes for supercapacitor applications. Chem Mater 2015, 27: 5314-5323.

[25] Peng C, Yang XF, Li YH, et al. Hybrids of two-dimensional $\mathrm{Ti}_{3} \mathrm{C}_{2}$ and $\mathrm{TiO}_{2}$ exposing $\{001\}$ facets toward enhanced photocatalytic activity. ACS Appl Mater Interfaces 2016, 8: 6051-6060.

[26] Halim J, Cook KM, Naguib M, et al. X-ray photoelectron spectroscopy of select multi-layered transition metal carbides (MXenes). Appl Surf Sci 2016, 362: 406-417.

[27] Cheng RF, Hu T, Hu MM, et al. MXenes induce epitaxial growth of size-controlled noble nanometals: A case study for surface enhanced Raman scattering (SERS). J Mater Sci Technol 2020, 40: 119-127.

[28] Zhou XZ, Huang X, Qi XY, et al. In situ synthesis of metal nanoparticles on single-layer graphene oxide and reduced graphene oxide surfaces. J Phys Chem C 2009, 113: 10842-10846.

[29] Li HY, Hou Y, Wang FX, et al. Flexible all-solid-state supercapacitors with high volumetric capacitances boosted by solution processable MXene and electrochemically exfoliated graphene. Adv Energy Mater 2017, 7: 1601847.

[30] Singh SK, Dhavale VM, Boukherroub R, et al. N-doped porous reduced graphene oxide as an efficient electrode material for high performance flexible solid-state supercapacitor. Appl Mater Today 2017, 8: 141-149.

[31] Tang J, Mathis TS, Kurra N, et al. Tuning the electrochemical performance of titanium carbide MXene by controllable in situ anodic oxidation. Angewandte Chemie 2019, 131: 18013-18019.

[32] Zhang B, Chen JD, Zhu H, et al. Facile and green fabrication of size-controlled AuNPs/CNFs hybrids for the highly sensitive simultaneous detection of heavy metal ions. Electrochimica Acta 2016, 196: 422-430.

[33] Atar N, Eren TJ, Yola ML, et al. Fe@Ag nanoparticles decorated reduced graphene oxide as ultrahigh capacity anode material for lithium-ion battery. Ionics 2015, 21: 3185-3192.

[34] Ghidiu M, Kota S, Halim J, et al. Alkylammonium cation intercalation into $\mathrm{Ti}_{3} \mathrm{C}_{2}$ (MXene): Effects on properties and ion-exchange capacity estimation. Chem Mater 2017, 29: 1099-1106.

[35] Ghidiu M, Lukatskaya MR, Zhao MQ, et al. Conductive two-dimensional titanium carbide 'clay' with high volumetric capacitance. Nature 2014, 516: 78-81.

[36] Mashtalir O, Lukatskaya MR, Kolesnikov AI, et al. The effect of hydrazine intercalation on the structure and capacitance of 2D titanium carbide (MXene). Nanoscale 2016, 8: 9128-9133.

[37] Boota M, Anasori B, Voigt C, et al. Pseudocapacitive electrodes produced by oxidant-free polymerization of pyrrole between the layers of 2D titanium carbide (MXene). 
Adv Mater 2016, 28: 1517-1522.

[38] Wen YY, Rufford TE, Chen XZ, et al. Nitrogen-doped $\mathrm{Ti}_{3} \mathrm{C}_{2} \mathrm{~T}_{x}$ MXene electrodes for high-performance supercapacitors. Nano Energy 2017, 38: 368-376.

[39] Zhao MQ, Ren CG, Ling Z, et al. Flexible MXene/carbon nanotube composite paper with high volumetric capacitance. Adv Mater 2015, 27: 339-345.

[40] Ren YY, Zhu JF, Wang L, et al. Synthesis of polyaniline nanoparticles deposited on two-dimensional titanium carbide for high-performance supercapacitors. Mater Lett 2018, 214: 84-87.

[41] Xu SK, Wei GD, Li JZ, et al. Binder-free $\mathrm{Ti}_{3} \mathrm{C}_{2} \mathrm{~T}_{x}$ MXene electrode film for supercapacitor produced by electrophoretic deposition method. Chem Eng J 2017, 317: 1026-1036.

[42] Zhou YH, Maleski K, Anasori B, et al. $\mathrm{Ti}_{3} \mathrm{C}_{2} \mathrm{~T}_{x}$ MXenereduced graphene oxide composite electrodes for stretchable supercapacitors. ACS Nano 2020, 14: 3576-3586.

[43] Liu Q, Yang JJ, Luo XG, et al. Fabrication of a fibrous $\mathrm{MnO}_{2} @ \mathrm{MXene} / \mathrm{CNT}$ electrode for high-performance flexible supercapacitor. Ceram Int 2020, 46: 11874-11881.

[44] Couly C, Alhabeb M, van Aken KL, et al. Asymmetric flexible MXene-reduced graphene oxide micro-supercapacitor. $A d v$ Electron Mater 2018, 4: 1700339.

[45] Xia QX, Fu JJ, Yun JM, et al. High volumetric energy density annealed-MXene-nickel oxide/MXene asymmetric supercapacitor. RSC Adv 2017, 7: 11000-11011.

[46] Lukatskaya MR, Mashtalir O, Ren CE, et al. Cation intercalation and high volumetric capacitance of twodimensional titanium carbide. Science 2013, 341: 15021505.

[47] Fan ZM, Wang YS, Xie ZM, et al. A nanoporous MXene film enables flexible supercapacitors with high energy storage. Nanoscale 2018, 10: 9642-9652.

Open Access This article is licensed under a Creative Commons Attribution 4.0 International License, which permits use, sharing, adaptation, distribution and reproduction in any medium or format, as long as you give appropriate credit to the original author(s) and the source, provide a link to the Creative Commons licence, and indicate if changes were made.

The images or other third party material in this article are included in the article's Creative Commons licence, unless indicated otherwise in a credit line to the material. If material is not included in the article's Creative Commons licence and your intended use is not permitted by statutory regulation or exceeds the permitted use, you will need to obtain permission directly from the copyright holder.

To view a copy of this licence, visit http://creativecommons. org/licenses/by/4.0/. 